\title{
Parâmetros nutricionais de dietas anunciadas na imprensa leiga destinada ao público masculino e feminino
}

\author{
Nutritional parameters of diets advertised in the lay press aimed at male and female public
}

\author{
Edeli Simioni de Abreu¹, Ana Carolina Almada Colucci Paternez¹, Daniela Maria \\ Alves Chaud², Fabiana Valverde ${ }^{3}$, Juliana Salgado Gaze ${ }^{3}$
}

\begin{abstract}
RESUMO
Objetivo: Analisar parâmetros nutricionais de dietas genéricas anunciadas na imprensa leiga destinada ao público masculino e feminino.

Materiais e Métodos: Estudo observacional descritivo. Quatro publicações destinadas ao público feminino e duas destinadas ao público masculino foram selecionadas, em função da periodicidade, tiragem, número de leitores e anos de publicação. Essas publicações foram acompanhadas durante um ano, no período de julho de 2009 a junho de 2010. Foram avaliados carboidratos, proteínas, lipídeos, cálcio, ferro, vitamina C, vitamina A, vitamina E, e o colesterol, além de informações sobre a duração da dieta, ingestão de líquidos, atividade física e dieta de manutenção.

Resultados: A duração das dietas variou entre 1 e 10 dias, pouco foi encontrado a respeito da utilização de líquidos e dieta de manutenção. Quanto à atividade física, houve ênfase somente em uma das revistas masculinas. Notou-se uma grande variação de conteúdo energético e de macro e micronutrientes entre as dietas analisadas, com a maior inadequação relacionada aos carboidratos. O valor calórico médio para as revistas femininas e masculinas foi, respectivamente, $1113 \pm 151$ e $1727 \pm 179$ kcal. Observou-se uma oferta deficiente dos micronutrientes analisados, destacando-se as deficiências na oferta de cálcio, ferro e vitaminas $A$ e $E$.

Conclusão: As dietas publicadas em imprensa leiga apresentam curta duração e vem, somente em alguns casos, acompanhadas de informações sobre a prática de atividade física e ingestão de líquidos. Em relação ao valor nutritivo, as mesmas apresentam um reduzido aporte de energia e nutrientes, especialmente de vitaminas e minerais, se adotadas por longo período de tempo.
\end{abstract}

Palavras-chave: dieta; perda de peso; valor nutritivo; publicações periódicas; comportamento alimentar.

\begin{abstract}
Objective: To analyze nutritional parameters of generic diets advertised in the press intended for male and female audience.

Materials and Methods: This was a descriptive observational study. Four publications targeted to women and two for male public were selected based on the periodicity, circulation, readership and publication years. These publications were analyzed for one year, from July 2009 to June 2010. The nutrients evaluated were: carbohydrates, lipids, proteins, calcium, iron, vitamin C, vitamin A, vitamin E, and cholesterol, as well as information about the duration of the diet, fluid intake, physical activity and diet maintenance.

Results: The duration of the diets were between 1 and 10 days and not much has been found concerning the use of fluids and maintenance diet. As for physical activity, emphasis was placed only on a male magazine. It was noted a wide variation in the energy content, as well as macronutrient and micronutrient between the diets analyzed, with the greatest inadequacy related to carbohydrates. The average caloric value to women's magazines and men were, respectively, $1113 \pm 151$ and $1727 \pm 179 \mathrm{kcal}$. There was a shortfall of the micronutrients analyzed, highlighting the deficiencies in the supply of calcium, iron and vitamins $A$ and $E$.

Conclusions: Diets published in the lay press present short duration and come, only in some cases, accompanied by information on physical activity and fluid intake. In relation to the nutritional value, they present a reduced intake of energy and nutrients, particularly vitamins and minerals, if taken for a long period of time.
\end{abstract}

Keywords: diet; weight loss; nutritive value; periodicals; feeding behavior.

\footnotetext{
${ }^{1}$ Nutricionista. Mestre e Doutora em Saúde Pública pela Universidade de São Paulo (USP). Docente dos cursos de Nutrição e Tecnologia em Gastronomia da Universidade Presbiteriana Mackenzie.

${ }^{2}$ Nutricionista. Mestre e Doutora em Pediatria e Ciências Aplicadas à Pediatria pela Universidade Federal de São Paulo. Docente do curso de Nutrição da Universidade Presbiteriana Mackenzie.

${ }^{3}$ Nutricionista graduada pela Universidade Presbiteriana Mackenzie.
} 


\section{INTRODUÇÃO}

A obesidade é uma doença crônica multifatorial e vem atingindo níveis alarmantes não somente no Brasil, mas em todo o mundo, em todas as classes socioeconômicas e faixas etárias ${ }^{1,2}$. Sob outra ótica, a obesidade, enquanto estigmatizadora, produz discriminação, preconceito e exclusão social ${ }^{3}$.

A obesidade e a crescente preocupação com um padrão estético se tornaram assunto de interesse em vários meios de comunicação em massa, consultados pela população leiga. Este panorama está presente em diversos países, como Estados Unidos ${ }^{4}$, Canadá ${ }^{5}$ e Inglaterra ${ }^{6}$. As principais formas pelas quais os meios de comunicação abordam esse tema são o estímulo à venda de produtos para emagrecimento e recomendações de dietas hipocalóricas. Nestas publicações podem-se encontrar mensagens que incitam o consumidor ao seguimento de dietas restritivas em calorias ou outras práticas, com o objetivo de redução de peso em pouco tempo e sem esforço ${ }^{7}$. Assim, a mídia não se limita a ser imparcial e apenas informar, mas também se posiciona para o que parece ser conveniente e lucrativo ${ }^{8}$.

Paradoxalmente, a mídia influencia o consumo de alimentos gordurosos e hipercalóricos, enquanto a sociedade valoriza a magreza. Este padrão de beleza influencia cada vez mais adolescentes e adultos, resultando em insatisfação de imagem corporal. Este cenário, por sua vez, favorece a adesão a dietas restritivas e a prática excessiva de exercícios físicos, com o objetivo de atingir um ideal corporal ${ }^{9}$.

No sexo masculino, o padrão corporal desejado também sofreu modificações ao longo do tempo ${ }^{10}$, surgindo um maior número de publicações direcionadas a esse público, contendo informações sobre treinos e dietas para aumento de massa muscular ${ }^{11}$. Pope et al. ${ }^{12}$, em pesquisa realizada com a população americana, demonstraram que os homens estão ultrapassando as mulheres no nível de insatisfação corporal. A preocupação com a imagem corporal, antes predominantemente feminina, passa a ser preocupação de ambos os gêneros ${ }^{13}$.

Nesse contexto, a publicação de dietas sem embasamento científico em nutrição, que podem causar malefícios aos indivíduos, tem sido objeto de preocupação da comunidade científica. Considerandose a mudança de perfil epidemiológico, com aumento da prevalência de obesidade, e o crescente aumento de informações veiculadas pela mídia, objetivou-se analisar parâmetros nutricionais de dietas genéricas anunciadas na imprensa leiga destinada ao público masculino e feminino.

\section{MATERIAIS E MÉTODOS}

Trata-se de um estudo observacional descritivo sobre dietas genéricas anunciadas pela imprensa leiga. Foram selecionadas quatro publicações destinadas ao público feminino e duas destinadas ao público masculino, em função da periodicidade, tiragem, número de leitores e anos de publicação, de acordo com dados de pesquisas realizadas pelo Instituto Verificador de Circulação.

Essas publicações foram acompanhadas durante um ano, para que fosse possível obter um ciclo completo com as quatro estações climáticas, no período de julho de 2009 a junho de 2010. Inicialmente, em cada exemplar das revistas, foram identificadas todas as dietas publicadas. Para cada dieta foi realizada análise crítica prévia dos alimentos e preparações mencionadas e, para padronização das quantidades e receitas, foram utilizadas as referências de Pinheiro et al. ${ }^{14}$ e de Fisberg e Slater ${ }^{15}$.

A análise da composição nutricional das dietas foi realizada com a utilização do programa AVANUTRI, versão $4.0^{16}$. Os parâmetros nutricionais avaliados foram conteúdo energético (kcal), carboidratos (\% do valor energético total da dieta - VET), proteínas (\% VET), lipídeos (\% VET), cálcio (mg), ferro (mg), vitamina C (mg), vitamina A (mg), vitamina $E(\mathrm{mg})$ e colesterol (mg). O critério adotado para seleção destes nutrientes foi a importância dos mesmos numa alimentação balanceada e as consequências que sua carência ou

TABELA 1 - Recomendações nutricionais de macronutrientes para indivíduos adultos.

\begin{tabular}{ccc}
\hline $\begin{array}{c}\text { Carboidrato } \\
(\% \text { VET) }\end{array}$ & $\begin{array}{c}\text { Proteína } \\
(\% \text { VET) }\end{array}$ & $\begin{array}{c}\text { Lipídeos } \\
\text { (\% VET) }\end{array}$ \\
\hline $45-65$ & $10-35$ & $20-35$ \\
\hline
\end{tabular}

Nota: VET - Valor energético total

Fonte: Institute of Medicine21 (2005) 
TABELA 2 - Recomendações nutricionais de micronutrientes para indivíduos adultos.

\begin{tabular}{|c|c|c|c|c|c|c|c|c|c|c|}
\hline \multirow[b]{2}{*}{ Sexo/faixa etária } & \multicolumn{2}{|c|}{ Cálcio } & \multicolumn{2}{|c|}{ Ferro } & \multicolumn{2}{|c|}{ Vitamina A } & \multicolumn{2}{|c|}{ Vitamina C } & \multicolumn{2}{|c|}{ Vitamina E } \\
\hline & $\begin{array}{l}\text { EAR } \\
\mathrm{mg} / \mathrm{d}\end{array}$ & $\begin{array}{c}\mathrm{UL} \\
\mathrm{mg} / \mathrm{d}\end{array}$ & $\begin{array}{l}\text { EAR } \\
\mathrm{mg} / \mathrm{d}\end{array}$ & $\begin{array}{c}\mathrm{UL} \\
\mathrm{mg} / \mathrm{d}\end{array}$ & $\begin{array}{l}\text { EAR } \\
\mathrm{mg} / \mathrm{d}\end{array}$ & $\begin{array}{c}\mathrm{UL} \\
\mathrm{mg} / \mathrm{d}\end{array}$ & $\begin{array}{c}\text { EAR } \\
\mathrm{mg} / \mathrm{d}\end{array}$ & $\begin{array}{c}\mathrm{UL} \\
\mathrm{mg} / \mathrm{d}\end{array}$ & $\begin{array}{l}\text { EAR } \\
\mathrm{mg} / \mathrm{d}\end{array}$ & $\begin{array}{c}\mathrm{UL} \\
\mathrm{mg} / \mathrm{d}\end{array}$ \\
\hline \multicolumn{11}{|l|}{ Masculino } \\
\hline $19-30$ anos & 800 & 2500 & 6 & 45 & 625 & 3000 & 75 & 2000 & 12 & 1000 \\
\hline $31-50$ anos & 800 & 2500 & 6 & 45 & 625 & 3000 & 75 & 2000 & 12 & 1000 \\
\hline \multicolumn{11}{|l|}{ Feminino } \\
\hline $19-30$ anos & 800 & 2500 & 8,1 & 45 & 500 & 3000 & 60 & 2000 & 12 & 1000 \\
\hline $31-50$ anos & 800 & 2500 & 8,1 & 45 & 500 & 3000 & 60 & 2000 & 12 & 1000 \\
\hline
\end{tabular}

EAR - Estimated Average Requirement. UL - Tolerable Upper Intake Level. Fonte: Institute of Medicine ${ }^{18-20,22}$ (2000, 2001, 2002, 2011).

excesso podem causar à saúde humana. Foi analisada a composição de vitamina $\mathrm{C}$ devido ao importante papel desse nutriente na absorção de ferro ${ }^{17}$.

A composição energética foi identificada pelo cálculo de calorias fornecidas por carboidratos (4 kcal/g), proteínas $(4 \mathrm{kcal} / \mathrm{g})$ e lipídeos $(9 \mathrm{kcal} / \mathrm{g})$. Para análise da adequação das dietas foram utilizadas as recomendações do Institute of Medicine (IOM) ${ }^{18-22}$, denominadas DRIs - Dietary Reference Intake. Os macronutrientes foram avaliados pela AMDR Acceptable Macronutrient Distribution Range ${ }^{21}$ (IOM, 2005), tabela 1.

No caso dos micronutrientes - ferro, cálcio, vitamina $A$, vitamina $C$ e vitamina $E$ - foi calculada, para cada dieta, a adequação aparente da oferta, adotando-se o EAR - Estimated Average Requirement - como ponto de corte, conforme proposto por Beaton ${ }^{23}$, utilizando-se a seguinte fórmula:

$$
z=D / D P_{D}=\frac{\bar{\gamma}-E A R}{\sqrt{V_{\text {nec }}=\left(V_{\text {int }} / n\right)}}
$$

Onde:

$Z=$ escore $-Z$

$D=$ diferença entre a média da dieta recomendada e a

EAR

DPD = desvio-padrão de $\mathrm{D}$

$\bar{\gamma}=$ média de nutrientes de $\mathrm{n}$ dias da dieta recomendada

$\mathrm{V}_{\text {nec }}=$ variância da necessidade

$\mathrm{V}_{\text {int }}=$ variância intrapessoal

$\mathrm{n}=$ número de dias indicados para seguimento da dieta
Para fins de cálculo, foram consideradas as recomendações nutricionais para homens e mulheres, com idade entre 19 e 50 anos (tabela 2). Considerando ser necessária a informação de um padrão habitual de consumo, considerou-se para o cálculo somente as dietas com dois ou mais dias de cardápio. Adotou-se como ponto de corte a confiabilidade de adequação de $70 \%{ }^{24}$. Assim, considerou-se dieta adequada aquela em que a oferta de micronutrientes calculada apresentou confiabilidade de adequação maior que $70 \%$. Foi avaliada a probabilidade da dieta ultrapassar níveis máximos de ingestão (UL: Tolerable Upper Intake Level). Neste caso, também foi adotado o ponto de corte de $70 \%$, considerando-se dieta inadequada aquela que apresentasse probabilidade maior de $70 \%$ de exceder o UL.

Quanto ao colesterol, foram adotadas as recomendações dietéticas para a população em geral, preconizadas pela American Heart Association ${ }^{25}$.

Para as variáveis numéricas, os dados foram apresentados em formas de tabelas e/ou gráficos. Para descrição dos dados, medidas de tendência central (média e desvio-padrão) foram utilizadas.

Por não se tratar de pesquisa envolvendo seres humanos, direta ou indiretamente, o presente projeto não foi submetido ao Comitê de Ética em Pesquisa.

\section{RESULTADOS}

Foram analisadas quatro publicações destinadas ao público feminino, nas quais havia a publicação de diversas dietas para perda de peso. Nas duas revistas destinadas ao público masculino, somente em uma 
TABELA 3 - Duração das dietas, ingestão de líquidos e incentivo à prática de atividade física de dietas publicadas em imprensa leiga.

\begin{tabular}{ccccccc}
\hline \multirow{2}{*}{$\begin{array}{c}\text { Revista/ } \\
\text { público-alvo* }\end{array}$} & $\begin{array}{c}\text { Duração } \\
\text { média (dias) }\end{array}$ & \multicolumn{2}{c}{ Ingestão de líquidos } & \multicolumn{2}{c}{ Atividade física } & Total \\
\cline { 3 - 5 } & & $\mathbf{S i m}$ & $\mathbf{N a ̃ o}$ & $\mathbf{S i m}$ & $\mathbf{N a ̃ o}$ & \\
$1(\mathrm{~F})$ & 2,6 & $\mathbf{n}(\%)$ & $\mathbf{n}(\%)$ & $\mathbf{n}(\%)$ & $\mathbf{n}(\%)$ & \\
$2(\mathrm{~F})$ & 6,1 & $0(0)$ & $25(100,0)$ & $8(17,6)$ & $28(82,3)$ & 34 \\
$3(\mathrm{~F})$ & 4,2 & $1(8,3)$ & $11(91,6)$ & $1(8,0)$ & $17(68,0)$ & 25 \\
$4(\mathrm{~F})$ & 6,2 & $0(0)$ & $12(100,0)$ & $0(0)$ & $11(91,6)$ & 12 \\
$5(\mathrm{M})$ & 2,6 & $0(0)$ & $3(100,0)$ & $2(66,6)$ & $1(33,3)$ & 3 \\
\hline
\end{tabular}

$\mathrm{F}=$ feminino. $\mathrm{M}=$ masculino

delas (revista 5) foi identificada informação referente à dieta. A sexta publicação selecionada apresentou somente orientações nutricionais breves, receitas de preparações e harmonização de alimentos e bebidas em relação ao assunto em estudo, não havendo dieta a ser calculada.

Nas seis revistas avaliadas foram identificadas 86 dietas. Foram analisadas 83 dietas publicadas nas revistas destinadas ao público feminino e 3 nas destinadas ao público masculino. Grande parte delas $(\mathrm{n}=24)$ fazia menção a dietas realizadas por personalidades de mídia, ou seja, continham exemplos de planos alimentares supostamente seguidos por pessoas famosas, sendo em sua grande maioria mulheres.

As revistas avaliadas ainda publicaram, além dos planos alimentares contendo a descrição dos alimentos e preparações a serem consumidos e suas respectivas quantidades, informações adicionais que têm por objetivo favorecer a motivação e otimizar os resultados a serem obtidos. A tabela 3 apresenta informações sobre a duração das dietas, ingestão de líquidos e incentivo à prática de atividade física, segundo publicação.

No que se refere ao valor energético, as dietas das revistas femininas e masculinas apresentaram, em média, $1113 \pm 151 \mathrm{kcal}$ e $1727 \pm 179 \mathrm{kcal}$ (tabela 4), respectivamente. A figura 1 apresenta a distribuição percentual da adequação de carboidratos, proteínas e lipídios aos intervalos recomendados pelo IOM ${ }^{11}$.

\section{DISCUSSÃO}

A maior parte das dietas recomendava o consumo de alimentos para fins especiais, com denominações diet, light, low e free. No entanto, tais produtos também apresentam relativo valor calórico e, por este motivo, sua ingestão deve orientada de maneira correta ${ }^{26}$. Montes de $\mathrm{Oca}^{27}$, em um estudo realizado com revistas semanais sobre saúde, destinada ao público leigo, determinou a tendência de informações sobre saúde, destacando-se o fato de temas relacionados à nutrição estarem atreladas à publicidade de produtos industrializados, o que evidencia a perda de imparcialidade dos artigos publicados nessas revistas.

Em relação à orientação sobre a ingestão de líquidos na dieta, observou-se que apenas uma pequena parcela das publicações apresentava tal indicação. As recomendações de ingestão de água variam de acordo com diferentes órgãos de saúde. No Guia Alimentar para a População Brasileira ${ }^{28}$ indica-se um método prático para calcular a necessidade de água para indivíduos adultos, que consiste em considerar o consumo de $1 \mathrm{~mL} / \mathrm{kcal}$ de energia gasta em condições moderadas de gasto energético e temperaturas ambientais não muito elevadas. De modo geral, recomenda-se a ingestão de pelo menos 2 litros (6 a 8 copos) de água por $\mathrm{dia}^{29}$, sendo que todas as revistas que indicaram o consumo de líquidos utilizaram essa recomendação como referência nas publicações.

Foi observada restrita orientação à prática de atividade física nas dietas publicadas em revistas femininas. No entanto, em uma das revistas masculinas analisadas, notou-se grande ênfase à importância da

TABELA 4. Contribuição percentual dos macronutrientes ao valor energético total (\% VET) de dietas publicadas em imprensa leiga, segundo sexo.

\begin{tabular}{lcccc}
\hline Sexo & $\begin{array}{c}\text { Energia } \\
\text { (kcal) }\end{array}$ & $\begin{array}{c}\text { Carboidrato } \\
\text { (\%VET) }\end{array}$ & $\begin{array}{c}\text { Proteína } \\
\text { (\%VET) }\end{array}$ & $\begin{array}{c}\text { Lipídio } \\
\text { (\%VET) }\end{array}$ \\
\hline Feminino & 1113 & 48,9 & 21,4 & 29,5 \\
Masculino & 1727 & 47,3 & 26,0 & 26,5 \\
\hline
\end{tabular}




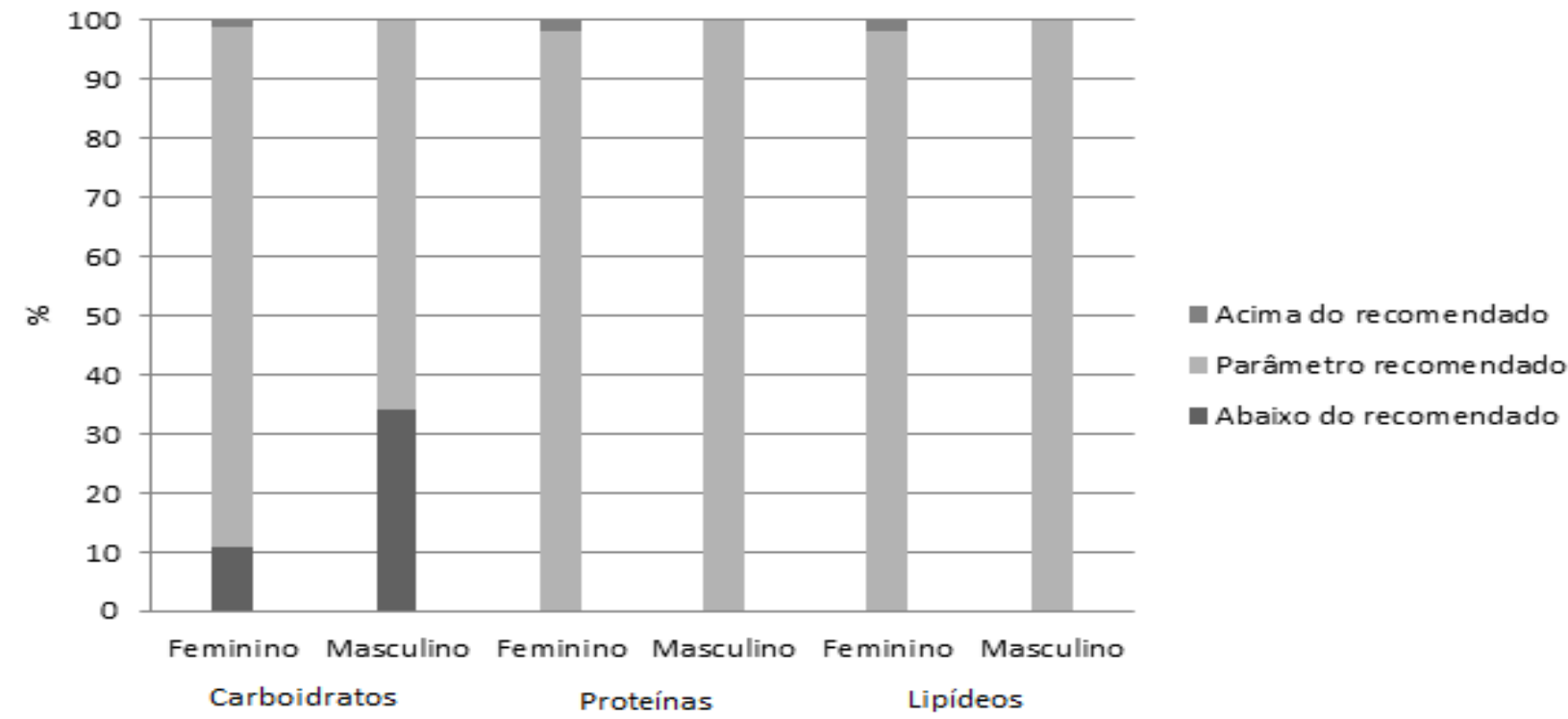

FIGURA 1 - Distribuição percentual da adequação da oferta de carboidratos, proteínas e lipídeos em relação ao valor energético total de dietas publicadas em imprensa leiga.

atividade física associada à adoção de uma dieta hipocalórica. Embora a prática de exercícios físicos seja reconhecidamente benéfica, devem ser considerados para a sua realização o tipo de exercício, a sua duração e frequência, que devem ser acompanhados por um profissional da área, uma vez que a realização de exercícios pode causar lesões se conduzida de maneira incorreta ${ }^{30}$.

Além das informações mencionadas, também é importante apontar a presença de dados relacionados ao consumo de bebidas alcoólicas, especialmente nas revistas voltadas ao público masculino, que tendem a incentivar em seus artigos a ingestão de alguns tipos de bebidas fermentadas e até mesmo de destilados. Contudo, o incentivo ao consumo de bebidas alcoólicas deve ser evitado, uma vez que a sua ingestão, em excesso, pode levar ao desenvolvimento de determinadas deficiências nutricionais, constituindo também como um fator de risco para o desenvolvimento de doenças crônicas não-transmissíveis, que representam a principal causa de morte da atualidade ${ }^{31}$.

Com relação ao tempo de duração da dieta, observou-se no presente estudo períodos que variaram entre 1 e 10 dias, o que diverge do observado no estudo de Amâncio e Chaud ${ }^{32}$, que não observaram a indicação de duração das dietas analisadas. De modo geral, a duração média das dietas publicadas é pequena, sendo a maior média identificada na revista 4 (6,2 dias). A intervenção que considera perda ponderal em curto período de tempo é considerada inadequada e insuficiente, pois pode não atingir o sucesso na manutenção do peso ${ }^{33}$.

Em relação ao valor energético das dietas foram identificados valores reduzidos, fato confirmado por estudos similares realizados por Amâncio e Chaud ${ }^{32}$, Souza $^{34}$ e Souza et al. ${ }^{35}$. Quando analisado o valor nutritivo médio por revista, observou-se que as revistas 2 e 4 (ambas para o público feminino) foram as publicações que continham dietas com menor valor energético médio. Apesar da contribuição percentual média dos macronutrientes ao valor energético estarem de acordo com relação às recomendações do $10 \mathrm{M}^{21}$, observou-se tendência de desequilíbrio, pois a maioria das dietas publicadas apresentou oferta elevada de proteínas, em detrimento à oferta de carboidratos.

A oferta de carboidratos foi abaixo do recomendado em $10,8 \%$ das dietas voltadas para o público feminino e em $33,3 \%$ das dietas para o público masculino. Apesar de exercerem papel fundamental na sobrevivência humana, por seu elevado conteúdo energético, ainda se observa, entre os indivíduos que objetivam a perda de peso e entre praticantes de atividade física, especialmente do sexo masculino, resistência ao consumo deste nutriente. A alimentação adequada em 
TABELA 5 - Valor nutritivo médio de dietas publicadas em imprensa leiga, segundo revista.

\begin{tabular}{|c|c|c|c|c|c|c|c|c|c|c|c|}
\hline $\begin{array}{l}\text { Revista/ } \\
\text { Público } \\
\text { alvo }\end{array}$ & $\begin{array}{c}\text { Energia } \\
\text { (kcal) }\end{array}$ & $\begin{array}{l}\text { Carb } \\
(\mathrm{g})\end{array}$ & $\begin{array}{l}\text { Prot } \\
\text { (g) }\end{array}$ & $\begin{array}{l}\text { Lip } \\
\text { (g) }\end{array}$ & $\begin{array}{l}\text { Fibras } \\
\text { (g) }\end{array}$ & $\begin{array}{l}\text { Cálcio } \\
(\mathrm{mg})\end{array}$ & $\begin{array}{l}\text { Ferro } \\
(\mathrm{mg})\end{array}$ & $\begin{array}{l}\text { Vit C } \\
(\mathrm{mg})\end{array}$ & $\begin{array}{l}\text { Vit A } \\
\text { (mg) }\end{array}$ & $\begin{array}{l}\text { Vit E } \\
\text { (mg) }\end{array}$ & $\begin{array}{c}\text { Colest } \\
(\mathrm{mg})\end{array}$ \\
\hline $1(F)$ & $\begin{array}{c}1780,9 \pm \\
169,7\end{array}$ & $\begin{array}{c}190,3 \pm \\
12,3\end{array}$ & $\begin{array}{c}68,4 \pm \\
21,0\end{array}$ & $\begin{array}{c}82,9 \pm \\
9,5\end{array}$ & $\begin{array}{c}19,9 \pm \\
1,8\end{array}$ & $\begin{array}{c}533,8 \pm \\
118,9\end{array}$ & $\begin{array}{c}22,5 \pm \\
3,1\end{array}$ & $\begin{array}{c}124,4 \pm \\
67,1\end{array}$ & $\begin{array}{c}490,3 \pm \\
189,5\end{array}$ & $\begin{array}{c}4,6 \pm \\
1,3\end{array}$ & $\begin{array}{c}161,2 \pm \\
99,3\end{array}$ \\
\hline $2(F)$ & $\begin{array}{c}868,8 \pm 1 \\
36,3\end{array}$ & $\begin{array}{c}110,6 \pm \\
10,1\end{array}$ & $\begin{array}{c}52,8 \pm \\
21,1\end{array}$ & $\begin{array}{c}23,9 \pm \\
4,8\end{array}$ & $\begin{array}{c}10,9 \pm \\
3,1\end{array}$ & $\begin{array}{c}367,4 \pm \\
89,5\end{array}$ & $\begin{array}{c}15,3 \pm \\
1,9\end{array}$ & $\begin{array}{c}150,7 \pm \\
106,5\end{array}$ & $\begin{array}{c}488,8 \pm \\
270,4\end{array}$ & $\begin{array}{c}4,1 \pm \\
2,4\end{array}$ & $\begin{array}{c}138,2 \pm \\
49,4\end{array}$ \\
\hline $3(F)$ & $\begin{array}{c}1280,0 \pm \\
170,7\end{array}$ & $\begin{array}{c}184,2 \pm \\
32,9\end{array}$ & $\begin{array}{c}69,2 \pm \\
11,2\end{array}$ & $\begin{array}{c}29,6 \pm \\
10,0\end{array}$ & $\begin{array}{c}15,8 \pm \\
4,0\end{array}$ & $\begin{array}{c}456,1 \pm \\
145,3\end{array}$ & $\begin{array}{c}8,4 \pm \\
1,8\end{array}$ & $\begin{array}{c}110,0 \pm \\
73,7\end{array}$ & $\begin{array}{c}381,5 \pm \\
125,4\end{array}$ & $\begin{array}{c}3,9 \pm \\
2,3\end{array}$ & $\begin{array}{c}168,2 \pm \\
31,6\end{array}$ \\
\hline $4(F)$ & $\begin{array}{c}842,1 \pm \\
129,2\end{array}$ & $\begin{array}{c}106,3 \pm \\
31,4\end{array}$ & $\begin{array}{c}59,9 \pm \\
17,3\end{array}$ & $\begin{array}{c}19,7 \pm \\
11,2\end{array}$ & $\begin{array}{c}12,0 \pm \\
3,8\end{array}$ & $\begin{array}{c}314,2 \pm \\
205,5\end{array}$ & $\begin{array}{c}8,9 \pm \\
6,3\end{array}$ & $\begin{array}{c}134,2 \pm \\
67,2\end{array}$ & $\begin{array}{c}366,1 \pm \\
308,8\end{array}$ & $\begin{array}{c}4,2 \pm \\
1,9\end{array}$ & $\begin{array}{l}98,4 \pm \\
109,2\end{array}$ \\
\hline $5(\mathrm{M})$ & $\begin{array}{c}1727,2 \pm \\
178,5\end{array}$ & $\begin{array}{c}204,5 \pm \\
30,2\end{array}$ & $\begin{array}{c}112,5 \pm \\
20,8\end{array}$ & $\begin{array}{c}51,0 \pm \\
7,9\end{array}$ & $\begin{array}{c}13,7 \pm \\
5,8\end{array}$ & $\begin{array}{c}912,4 \pm \\
298,3\end{array}$ & $\begin{array}{l}9,7 \pm \\
96,8\end{array}$ & $\begin{array}{c}148,5 \pm \\
51,9\end{array}$ & $\begin{array}{c}876,7 \pm \\
283,4\end{array}$ & $\begin{array}{c}12,4 \pm \\
1,4\end{array}$ & $\begin{array}{c}441,9 \pm \\
87,5\end{array}$ \\
\hline
\end{tabular}

termos de oferta de carboidratos contribui para a manutenção do peso corporal e a adequada composição corporal, contribuindo para a manutenção da saúde s0,36 $^{30}$

Por sua vez, $2,4 \%$ das dietas femininas se mostraram acima do recomendado em relação à oferta de proteínas, enquanto que a totalidade das dietas para o público masculino se mostraram adequadas. Cabe salientar que a contribuição percentual média de proteínas ao valor energético total foi mais elevada nas dietas para o público masculino. Ainda, o elevado percentual de adequação das dietas decorre do amplo intervalo recomendado de contribuição deste nutriente $(10-35 \%$ VET). Se fossem considerados outros parâmetros referenciais, como os da World Health Organization $^{37}$, que preconiza entre 10 e $15 \%$, ou ainda da Sociedade Brasileira de Alimentação e Nutrição ${ }^{38}$, que recomenda a ingestão de 10 a $12 \%$ de proteínas, o percentual de dietas inadequadas seria maior.

De modo geral, a avaliação da adequação de micronutrientes das dietas voltadas para o público feminino aponta elevado percentual de inadequação, caracterizado por conteúdo insuficiente de cálcio, ferro, vitamina A e vitamina E. Somente a vitamina C, nutriente amplamente disponível em frutas cítricas, apresentou-se suficiente em $82 \%$ das dietas analisadas. Inegavelmente, este panorama decorre do reduzido conteúdo energético de tais dietas, o que limita, por conseqüência, a oferta dos demais nutrientes. Considerando-se que para os micronutrientes somente foi possível avaliar duas dietas para o sexo masculino, inferências a respeito de tais dados são limitadas.

Souza et al. ${ }^{35}$, ao analisarem parâmetros nutricionais de dietas de emagrecimento veiculadas por revistas não-científicas, detectaram insuficiência na oferta de vitamina A e cálcio, da mesma forma que o presente estudo. Outro estudo sobre o mesmo tema, realizado na Universidade Federal de São Paulo, demonstrou inadequação para cálcio, ferro e vitaminas $A$ e $E^{39}$. Ao se analisar o risco de consumo excessivo atentando-se aos limites máximos toleráveis (UL), nenhuma dieta analisada mostrou-se inadequada.

A oferta média de colesterol das dietas foi de $138,5 \mathrm{mg}$ para o público feminino e de $242,0 \mathrm{mg}$ para o masculino. Tendo em vista que a recomendação, segundo a Sociedade Brasileira de Cardiologia ${ }^{40}$, é de um consumo inferior a 200mg por dia, as dietas para o público masculino apresentam elevada quantidade deste componente. Amâncio e Chaud $^{32}$, por sua vez, identificaram cerca de $10 \%$ de inadequação para colesterol nas dietas de revistas femininas.

Outro propósito do presente estudo foi observar se, nas publicações analisadas, há orientação de consumo de componentes fitoterápicos, porém não foram identificadas informações relevantes.

Os dados do presente estudo demonstraram que, de modo geral, as dietas publicadas em imprensa leiga apresentam curta duração e vem, somente em alguns casos, acompanhadas de informações sobre a prática de atividade física e ingestão de líquidos. No que se 
TABELA 6 - Adequação da oferta de micronutrientes de dietas publicadas em imprensa leiga, segundo sexo.

\begin{tabular}{lcccc}
\hline & \multicolumn{2}{c}{ Feminino $\mathbf{( n = 7 2 )}$} & \multicolumn{2}{c}{ Masculino (n=02) } \\
\cline { 2 - 5 } Nutriente & $\begin{array}{c}\text { Dieta } \\
\text { adequada } \\
\mathbf{n}(\%)\end{array}$ & $\begin{array}{c}\text { Dieta } \\
\text { inadequada } \\
\mathbf{n}(\%)\end{array}$ & $\begin{array}{c}\text { Dieta } \\
\text { adequada } \\
\mathbf{n}(\%)\end{array}$ & $\begin{array}{c}\text { Dieta } \\
\text { inadequada } \\
\mathbf{n}(\%)\end{array}$ \\
\hline Cálcio (mg) & $0(0)$ & $72(100,0)$ & $0(0)$ & $2(100,0)$ \\
Ferro (mg) & $14(19,4)$ & $58(80,6)$ & $2(100,0)$ & $0(0)$ \\
Vitamina C (mg) & $59(81,9)$ & $13(18,1)$ & $2(100,0)$ & $0(0)$ \\
Vitamina A (mg) & $4(5,6)$ & $68(94,4)$ & $2(100,0)$ & $0(0)$ \\
Vitamina E (mg) & $1(1,4)$ & $71(98,6)$ & $0(0)$ & $2(100,0)$ \\
\hline
\end{tabular}

refere ao valor nutritivo, conclui-se que as dietas publicadas apresentam um reduzido aporte de energia e nutrientes, especialmente de vitaminas e minerais, se adotadas por longo período de tempo. Mesmo admitindo as limitações do estudo, é importante enfatizar que a adoção de tais dietas pelo público leigo, sem a devida orientação de um profissional de saúde, especialmente o nutricionista, pode representar risco à saúde.

\section{REFERÊNCIAS}

1. Monteiro CA, D'A Benicio MH, Conde WL, Popkin BM. Shifting obesity trends in Brazil. Eur J Clin Nutr. 2000; 54:342-6.

2. Pinheiro ARO, Freitas SFT, Corso ACT. An epidemiological approach to obesity. Rev Nutr. 2004; 17:523-33.

3. Felippe FML. O peso social da obesidade. Encipecom [on line] 2004. Disponível em: http://encipecom.metodista.br/ mediawiki/index.php/O_pesosocialda_obesidade>. Acesso em: 22 jun. 2012.

4. American Diabetes Association. Americans: television is largest, but among least valued sources for nutrition information. Hot Topics [online] 1997. Disponível em <http://www.eatright.org/>. Acesso em: 20 mar. 2013.

5. Barton LA, Woolcott DM, Sheeshka JD, Tomasik HH. Readers' awareness and use of newspaper nutrition information. J Can Diet Assoc. 1997; 58:21-6.

6. Buttriss JL. Food and nutrition: attitudes, beliefs, and knowledge in the United Kingdom. Am J Clin Nutr. 1997; 65:1985S-95S

7. Hoff TMC, Felerico SP. Precisa-se de homens bonitos: a disciplinarização no discurso da beleza masculina na mídia impressa. In: Cunha KC. Em torno da masculinidade. São Paulo: Estação das Letras; 2008.

8. Guareschi P. Pressupostos psicossociais da exclusão: competitividade e culpabilização. In: Sawaia B. As artimanhas da exclusão. Petrópolis: Vozes; 1999.

9. Andrade A, Bosi MLM. Mídia e subjetividade: impacto no comportamento alimentar feminino. Rev Nutr. 2003; 16:117-25.

10. Camargo FC, Hoff TMC. Erotismo e mídia. São Paulo: Expressão e Arte; 2002.

11. Porto AA, Lins RG. Imagem corporal masculina e a mídia Lect Educ Física Deportes 2009;132:1.

12. Pope HG, Phillips KA. Olivardia R. O Complexo de Adônis. a obsessão masculina pelo corpo. Rio de Janeiro: Campus; 2000.
13. Ballone GJ. Vigorexia. [online] 2004. Disponível em: http://gballone.sites.uol.com.br/alimentar/vigorexia.html. Acesso em: 12 mar. 2010

14. Pinheiro ABV, Lacerda EMA, Benzecry EH, Gomes MCS, Costa VM. Tabela para avaliação de consumo alimentar em medidas caseiras. 5.ed. São Paulo: Atheneu; 2004.

15. Fisberg RM, Slater B. Manual de Receitas e Medidas Caseiras para Cálculo de Inquéritos Alimentares: manual elaborado para auxiliar o processamento de inquéritos alimentares. São Paulo: Signus; 2002.

16. Santana RI. Avanutri - Software de avaliação nutricional. Versão 4.0. Rio de Janeiro; 2009.

17. Tetens I, Larsen TM, Kristensen MB, Hels $O$, Jensen $M$, Morberg CM, Thomsen AD, Højgaard L, Henriksen M. The importance of dietary composition for efficacy of iron absorption measured in a whole diet that includes rye Bread fortified with ferrous fumerate: a radioisotope study in Young women. Br J Nutr. 2005;94(5):720-6.

18. IOM. Dietary Reference Intakes for Vitamin C, Vitamin E, Selenium and Carotenoids. Washington (DC): National Academy Press; 2000.

19. IOM. Dietary Reference Intakes: Applications in Dietary Assessment. Washington (DC): National Academy Press; 2001.

20. IOM. Dietary Reference Intakes for Vitamin A, Vitamin K, Arsenic, Boron, Chromium, Copper, lodine, Iron, Manganese, Molybdenum, Nickel, Silicon, Vanadium, and Zinc. Washington (DC): National Academy Press; 2002.

21. IOM. Dietary Reference Intakes for Energy, Carbohydrate, Fiber, Fat, Fatty Acids, Cholesterol, Protein, and Amino Acids (Macronutrients). Washington (DC): National Academy Press; 2005

22. IOM. Dietary Reference Intakes for Calcium and Vitamin D. Washington (DC): National Academy Press; 2011.

23. Beaton GH. Ingestão Dietética Recomendada: indivíduos e populações. In: Shils ME, Olson JÁ, Shike M, Ross AC. Tratado de nutrição moderna na saúde e na doença. Barueri: Manole; 2003

24. Fisberg RM, Marchioni DML, Slater B. Recomendações nutricionais. In: Fisberg RM, Slater B, Marchioni DML, Martini LA. Inquéritos alimentares: métodos e bases científicos. São Paulo: Manole; 2005.

25. American Heart Association. Dietary Recommendations. $2000 . \quad$ Disponível em: http://www.ameericanheartassociation.org. Acesso em: 20 mar. 2013.

26. Oliveira SP, Assumpção BV. Alimentos dietéticos: evolução do conceito, da oferta e do consumo. Hig Aliment. 2000;11:36-42.

27. Montes de Oca, A. La información en salud en revistas dominicales: ¿una vía para la publicidad indirecta? An Venez Nutr. 2004; 17:97-102. 
28. Brasil. Ministério da Saúde. Guia Alimentar para a População Brasileira. Brasília (DF): Ministério da Saúde 2005.

29. Brasil. Ministério da Saúde, Departamento de Atenção Básica, Secretaria de Atenção à Saúde. Política Nacional de Alimentação e Nutrição. 2.ed. Brasília: Ministério da Saúde; 2005.

30. Sociedade Brasileira de Medicina do Esporte. Diretrizes da Sociedade Brasileira de Medicina do Esporte. 2009. Rev Bras Med Esporte;15:3-12.

31. Kachani AT, Brasiliano S, Hochgraf PB. O impacto do consumo alcoólico no ganho de peso. Rev Psiq Clín. 2008; 35,supl 1;21-4.

32. Amancio OMS, Chaud DMA. Weight loss diets advertised in non-scientific publications. Cad Saúde Pública 2004; 20:1219-22.

33. Klack K, Carvalho JF. A importância da intervenção nutricional na redução do peso corpóreo em pacientes com síndrome do anticorpo antifosfolípide. Rev Bras Reumat. 2008; 48:134-40.

34. Souza DM. Revistas Femininas: História, Comunicação e Nutrição. Uma Análise Quantitativa e Qualitativa. 2005. Dissertação (Mestrado em Nutrição Humana) Universidade de São Paulo, São Paulo, 2005.

35. Souza EL, Pinto ICS, Lima MA, Targino DMG. Parâmetros nutricionais de dietas de emagrecimento, disponíveis em revistas não científicas impressas. 2006. Hig Aliment;20:27-33.
36. Egashira EM, Miziara APB, Leoni LAB. Grupo do arroz, pão, massa, batata e mandioca. In: Philippi ST (organizadora) Pirâmide dos alimentos: fundamentos básicos de nutrição. São Paulo: Manole, 2008.

37. World Health Organization. Obesity: preventing and managing the global epidemic. Report of a WHO consultation on obesity. Geneva; 1998.

38. Vannucchi H, Menezes EW, Campana AO, Lajolo FM. Avaliação das recomendações nutricionais adaptadas à população brasileira. Sociedade Brasileira de Alimentação e Nutrição (SBAN), 1990.

39. Di Giulio G. Dietas e revistas não-especializadas: uma combinação perigosa. 2005. Ciênc Cult;57:17.

40. Sociedade Brasileira de Cardiologia. IV Diretrizes brasileiras sobre dislipidemias e diretrizes de prevenção de aterosclerose do Departamento de Aterosclerose da Sociedade Brasileira de Cardiologia. Arq Bras Cardiol. 2007; 88(Supl. I):1-19.

\section{Endereço para correspondência}

Edeli Simioni de Abreu

Alameda São Caetano n` 857, apto. 43

São Caetano do Sul/SP - CEP 09560-051

Telefone: +55 1142217535

E-mail: edelisabreu@gmail.com 